\title{
A Meticulous Review of Diverse Etiologies and Pathophysiology of Anemia in Chronic Liver Disease
}

Nazanin Azadeh ( $\square$ nazaninazadeh91@gmail.com )

Shiraz University of Medical Sciences

Mohammad Azadeh

Novosibirsk State Medical University

\section{Research Article}

Keywords: Liver cirrhosis, Anemia, Portal Hypertension, Chronic Liver disease

Posted Date: March 2nd, 2022

DOI: https://doi.org/10.21203/rs.3.rs-1273896/v1

License: (c) (i) This work is licensed under a Creative Commons Attribution 4.0 International License. Read Full License 


\section{Abstract \\ Background}

Anemia in chronic liver diseases is a common condition with a wide variety of etiologic factors, some of which are life-threatening and need immediate evaluation and intervention. Furthermore, the severity of anemia has been associated with a poor disease prognosis and quality of life of patients. Herein, we have investigated the current literature regarding the spectrum of anemia in advanced liver diseases.

\section{Results}

Anemia of chronic disease, gastrointestinal bleeding, malnutrition, iron deficiency anemia and hypersplenism are the common etiologies of anemia accompanied by liver diseases. Medication side effects, viral-induced aplastic anemia and alcohol related hematologic abnormalities constitute the other parts of spectrum of anemia in liver diseases.

\section{Conclusion}

Among the various mechanisms of anemia in liver diseases, some are life-threatening and some can be controlled. Investigation of etiology of anemias in chronic liver diseases is recommended, as diseasespecific treatments will ameliorate the anemia and enhance patients' quality of life.

\section{Introduction}

Anemia is a common multifactorial finding in advanced liver diseases. Malnutrition, gastrointestinal bleeding, hypersplenism, drug side effect and viral aplastic anemia are considered as common etiologies of anemia in cirrhosis patients (1). Paternostro et al, have reported the prevalence of anemia to be $52.9 \%$ in cirrhosis patients, with $49.2 \%$ of which diagnosed as iron deficiency anemia (IDA). IDA is especially more prevalent in early stages of cirrhosis (2). In other studies the prevalence of anemia among chronic liver disease (CLD) patients have been reported up to two thirds of the patients (3). Anemia in liver cirrhosis is especially noteworthy as it is considered as a marker of disease severity and a prognostic factor. Singh et al, reported a decreasing trend in hemoglobin $(\mathrm{Hb})$ concentration as severity of cirrhosis increases. Thus, anemia has been introduced as a strong predictor of decompensation and poor outcome in end-stage liver disease in various studies (4-6). Anemia also contributes to low quality of life in CLD patients both before and after liver transplantation (7). However a common symptom, the diagnosis of etiologies of anemia in CLD is of utmost importance, as sometimes a life-threatening underlying condition may be present and specific treatments might be applied to alleviate the condition and increase patients' quality of life (8).

\section{Methods}


To retrieve the desired information, the current literature were surveyed by searching for anemia, chronic liver diseases, liver cirrhosis and portal hypertension as keywords in Google Scholar, PubMed and Scopus.

\section{A brief review of hemoglobin synthesis and pathophysiology of anemia}

A practical classification of anemia, classifies the condition to hypoproliferative anemias, when inadequate hemoglobin is produced and hemolytic anemias, when hemoglobin is prematurely destructed, either in vessels or spleen. Besides, in evaluation of anemia, blood loss should be considered as a potential etiology (9).

A tetramer of polypeptide globin chains, each bound to a heme group consist hemoglobin $(\mathrm{Hb})$. The heme group refers to a central ferrus iron ion $\left(\mathrm{Fe} 2^{+}\right)$surrounded by 4 porphyrin rings (10). Any condition interfereing with synthesis of either heme or globin chains will provoke hypoproliferative anemia. Nutritional deficiency or malabsorption of iron, vitamin B12 and folate, unbalanced hemoglobin synthesis in thalassemia syndromes, disturbed porphyrin synthesis in sideroblastic anemias, bone marrow disorders, renal failure, hypothyroidism, chronic diseases and liver disease constitute the spectrum of the most notable etiologies ultimately resulting in hypoproliferative anemia. Hemolytic anemia is divided into extravascular and intravascular hemolysis. Extravascular hemolysis occurs when the defective RBCs are trapped and destructed in the spleen. The defects might arise from hemoglobinopathies, enzymopathies and membranopathies which are more prevalently hereditary conditions (11). Immune hemolysis, microangipathic hemolytic conditions and infections cause RBCs to be lysed within blood vessels and result in intravascular hemolysis (12).

\section{Portal hypertension}

As liver fibrosis progresses in the course of cirrhosis, the vascular resistance of hepatic tissue increases, resulting in portal hypertension (13). Meanwhile, vasodilators including nitric oxide, prostaglandins and vasoactive intestinal peptide are produced to expand the splanchnic blood flow (14). Consequently portal vein dilates and veno-venous shunts form or re-open to maintain the blood flow in portal vein (15). The systemic vasodilatation and arterial under- filling stimulates volume-preserving mechanisms to retain fluid in the circulation. The activation of renin-angiotensin aldosterone and antidiuretic hormone contribute to a hyperdynamic circulatory state (16). When hepatic venous pressure gradient raises above $5 \mathrm{mmHg}$, gastrointestinal varices appear, the most troublesome of which are esophageal varices with high morbidity and mortality in case of bleeding (17). The pooled blood in spleen alongside with other mechanisms causes splenomegaly as another consequence of portal hypertension (18).

\section{A. Hemodilution}

Fluid retention causes hematocrit to be reported lower due to a dilutional mechanism. Such phenomenon happens in hypervolemic states including pregnancy, heart failure, excess fluid administration and of course, cirrhosis (19). Hence, an increase in plasma volume rather than true RBC mass reduction might be 
considered in interpreting the laboratory reports in the mentioned conditions. On the other hand, in response to chronic anemia, inhibition of basal endothelium-derived relaxing factor is reduced, leading to vasodilatation and neuro-hormonal compensatory sodium and water retention to increase the cardiac output (20). The anemia-induced hypervolemia, interestingly, exacerbates the hyperdynamic state caused by portal hypertension (21).

\section{B. Splenomegaly}

Destructing the old or diseased RBCs is one of the functions of spleen. Hypersplenism refers to the condition of spleen enlargement and one or more peripheral cytopenia in presence of normocellular bone marrow and returning of blood cell numbers to normal after splenectomy (22). The number of any of the blood cells can be affected by hypersplenism, with platelet as the most and RBC as the least sensitive ones (23). To date, various theories have been suggested as pathophysiology of cytopenia in hypersplenism. The most recognized theory associates the increased volume of blood passing the splenic tissue and consequent higher cell destruction (24). According to Shah et al, the phagocytic activity of spleen increases as its size enlarges during the course of cirrhosis leading to excess destruction of RBCs and anemia (25). Due to hypersplenism, in cirrhosis patients the hemoglobin concentration does not increase as effectively as normal population after RBC transfusion (26). Lv et al, reported $91 \%$ prevalence of anemia, either alone or in combination with other cytopenias in liver cirrhosis patients with splenomegaly (23). They reported the normalization of blood cell counts in $80.5 \%$ of cirrhosis patients after splenectomy in another study (27). Various studies have achieved promising outcomes after splenectomy in cirrhosis patients. Liver fibrosis progression, cytopenias and gastrointestinal bleeding have been ameliorated after the procedure. However, due to risks of operation and infections, splenectomy is not far and wide practiced $(28,29)$.

\section{Gastrointestinal bleeding}

Liver cirrhosis patients are a vulnerable group to gastrointestinal (GI) bleeding. Diminished production of coagulation factors in the liver diseases, as well as thrombocytopenia, subject the patients to bleeding tendency, which in addiction to portal hypertensive consequences increases the risk of bleeding in the $\mathrm{Gl}$ tract (30). The most catastrophic GI bleeding occurs in acute esophageal variceal bleeding, which needs urgent management to avoid mortality (31). Bleeding from portal hypertensive gastropathy and gastric vascular ectasia lesions most commonly manifest as chronic anemia in cirrhotic patients. Peptic ulcer is another etiology of chronic bleeding and anemia in this patient population, which forms a smaller proportion of chronic GI bleeding in cirrhosis (32). The most common cause of lower GI bleeding in cirrhosis is hemorrhoids with portal hypertensive colopathy as the second most common etiology. Khalifa et al, reported that $33 \%$ of lower GI bleeding in cirrhosis patients needed immediate resuscitation with blood products (33). Portal hypertensive enteropathy can be an obscure source of GI bleeding in cirrhosis (34).

\section{Aplastic anemia}


Hepatitis-associated aplastic anemia (HAAA) is a well-recognized and rare phenomenon, defined as pancytopenia and hypocellular marrow occurring two to three months after an acute hepatitis episode. The condition has been reported in all the hepatotropic virus infections including hepatitis A (HAV), B (HBV), C (HCV) ,D (HDV), E (HEV) and G (HGV) viruses (35). However, in most cases of HAAA no HAV, HBV, HCV association is found. The pancytopenia in HAAA can be extremely severe and sometimes lethal (36). Parvovirus B19 (B19V) and Epstein-Barr virus are other infectious etiologies of aplastic anemia. B19V is previously reported as an etiology of fulminant liver failure and concurrent aplastic anemia (37). The pathophysiology of HAAA is attributed to cytotoxic T cells (CD8+), which recognize hematopeitic and hepatic antigens as similar targets and when sensitized in an acute attack of hepatitis, they destruct the bone marrow cells as well. The process is mediated by gamma interferon (38).

\section{Alcoholic liver disease}

Alcohol contributes to anemia in various mechanisms besides the common anemia pathophysiology in general cirrhosis patients. Alcohol has direct toxic effect on bone marrow hematopoietic cells, manifesting as bone marrow suppression and pancytopenia which is reversible after abstinence from alcohol (39). Folate deficiency, either primarily when the patient replaces food with alcohol or secondary to alcohol's effects contributes to megaloblastic anemia. Alcohol decreases the serum level of folate by various mechanisms including increased urinary excretion of folate and decreasing its absorption in jejunum (40). Acetaldehyde, the product of alcohol oxidation in liver, increases the degradation rate of pyridoxal phosphate, the co-factor of aminolevulinic acid synthase enzyme. The enzyme catalyzes a step in heme synthesis. Thus, alcohol causes the defective production of heme and consequent accumulation of unutilized iron in mitochondria forming the so-called sideroblasts (41). Sideroblastic anemia is present in $25-30 \%$ of anemic heavy alcohol drinkers and is reversible in cases of alcohol abstinence (42). Sideroblastic anemia occurs in alcoholic patients who have concurrent folate deficiency (43). Another less common mechanism of anemia in alcoholic liver disease is the formation of acanthocytes (spur cells), which can be seen in end-stage liver diseases but is more frequently associated with heavy alcohol consumption (44). Abnormal lipid distribution in RBC membrane deforms the normal cells to acanthocytes. Acanthosis is present in up to $31 \%$ of advanced liver cirrhosis patients and is a predicting factor of mortality (45). The spur cells have defective membranes, making them susceptible to hemolysis when passing through spleen (46).

\section{Viral hepatitis}

Patients with viral-induced liver cirrhosis develop anemia with mechanisms common with other liver disease including malnutrition, GI bleeding and anemia of chronic disease, as well as unique etiologies attributed to the virus and its treatment (47). As earlier mentioned, rarely viral hepatitis attacks are followed by aplastic anemia in the recovery time, defined as HAAA.

A common therapy regimen for hepatitis $C$ is a combination of pegylated interferon and ribavirin. Ribavirin gets phosphorylated as cells uptake it and to exit the cell for elimination, the drug needs to de de-phosphorylated. As the RBCs lack the essential enzymes for dephosphorylation, the triphosphated 
Rivavirin molecules accumulate in the cell, making them susceptible to hemolysis (48). Other authors have suggested that the depletion of ATP during phosphorylation of Ribavirin leads to oxidative stress in RBCs and consequent hemolysis (49). The Interferon-induced hematopoietic stem cell suppression exacerbates the anemia caused by Ribavirin. It is reported that $20 \%$ of patients finally need Ribavirin dose reduction to alleviate anemia (50).

\section{Anemia of chronic disease, iron deficiency anemia and iron hemostasis}

The ferrous iron (Fe2+) is absorbed by enterocytes of duodenum and jejunum. To enter the blood, the ion is transported via the trans-membrane ferroportin exporter. Ferroportin also exists on membrane of ironstoring cells such as hepatocytes and macrophages (51). Iron is then, mainly transported by transferrin protein in the blood (52). As iron is toxic for the cells, it is stored bound to the ferritin protein in the cells. Hence, blood ferritin levels correlate with amount of iron storage in the body (53). Hepcidin, a peptide hormone produced in the liver, downregulates the release of iron from enterocytes and reticuloendothelial cytes by inhibiting ferroportin. Thus, hepcidin plays a crucial role in iron hemostasis (54).

In chronic inflammatory states like viral infections, organ rejection, inflammatory bowel disease, autoimmune conditions and tumors, an altered cytokine-mediated iron hemostasis results in impaired erythropoiesis. Numerous chronic liver diseases including viral hepatitis and autoimmune liver diseases share an inflammatory background. The pro-inflammatory cytokines including tumor necrosis factor (TNF- $a$ ) and interleukin-1, 6 and 10 are the major players to limit the iron availability to the erythrocyte progenitor cells and subsequent erythropoiesis. The changes occur by diminished erythropoietin production, inhibition of duodenal absorption of iron, reduced life span of RBCs, increased macrophage erythrophagocytosis, iron sequestration in macrophages and decreased release of the ion from macrophages (55). Furthermore, hepcidin is an acute phase reactant protein and is highly produced during inflammation, leading to reduced release of iron form enterocytes and macrophages (56). Various previous studies have reported increased expression of pro-inflammatory cytokines and hepcidin in nonalcoholic steatohepatitis (NASH). Obesity-induced inflammation and consequent high hepcidin levels promote to anemia of inflammation in $\mathrm{NASH}$, as the leading cause of chronic liver diseases throughout the globe (57). The anemia of chronic disease (ACD), also known as anemia of inflammation, is initially a mild to moderate normocytic normochromic anemia with decreased reticulocyte count and transferrin saturation level, the two characteristics shared with IDA. ACD and IDA are the leading causes of anemia in chronic liver diseases (3). IDA is a relatively common finding in this patient population which mainly is a result of acute or chronic blood loss from GI tract (58).

Diagnosis of co-existing IDA in chronic inflammation is of great importance, so iron supplementation and further investigation for potential sources of blood loss will be practiced to alleviate the anemia. Serum ferritin level, the indicator of iron storage, can be a useful laboratory parameter to differentiate IDA from ACD. Hepcidin can also be considered to differentiate the two conditions (56). However, diagnoses of ACD in presence of IDA is challenging. Ferritin is an acute phase reactant which despite presence of IDA, 
could be raised in inflammation, making it a less reliable test to differentiate ACD and ACD with concurrent IDA (59). Table 1. demonstrates the laboratory parameters in IDA and ACD.

Table 1. The laboratory parameter changes in IDA and ACD.

\begin{tabular}{|lllll|}
\hline Anemia etiology & Ferritin & Serum Iron & Transferrin saturation & Hepcidin \\
\hline IDA & Low & Low & Low & Low \\
\hline ACD & High & Low & Low & High \\
\hline IDA+ACD & Variable & Low & Low & Variable \\
\hline
\end{tabular}

\section{Bilirubin toxicity to erythrocytes}

The bilirubin's toxic effect on erythrocytes is a less investigated mechanism in anemia in CLD. Erythrocytes lack the major player of classic programmed cell death (apoptosis), namely nucleus and mitochondria. However, during oxidative stress, hyperosmolarity and several other insults, the erythrocytes undergo a suicidal death, called eryptosis (60). During eryptosis, an influx of $\mathrm{Ca}^{+}$ions and subsequent activation of $\mathrm{Ca}^{+}$-sensitive $\mathrm{K}^{+}$channels leads to depletion of cytosolic $\mathrm{K}^{+}$and $\mathrm{CL}^{-}$ions and cell shrinkage (61). Scrambling of cell membrane exposes phosphatidylserine to macrophages and the diseased RBCs are then recognized by the macrophages and undergo phagocytosis (62). Lang et al, have reported $\mathrm{k}+$ efflux, appearing of cell membrane phosphatidylcholine alongside with other manifestations of eryptosis in mice RBCs exposed to bilirubin. Then, they analyzed the number of RBCs undergoing accelerated cell death by detecting phosphatidylcholine as it binds with annexin V. A significant correlation of serum bilirubin levels and annexin V-bound RBCs was reported in the study. Further in vitro investigations, revealed increased number of annexin V-bound RBCs when exposed to serum of patients with cirrhosis and hyperbilirubinemia. Albumin was introduced as a protective factor against eryptosis as it binds bilirubin. Low serum albumin was consequently a contributing factor to premature death of RBCs in hyperbilirubinemic states (63). Acidosis is also an exacerbating factor of unconjugated bilirubin toxicity to erythrocytes (64).

\section{Other considerations}

In addition to common etiologies of anemia in CLD, exclusive causes might contribute to the condition, depending on the underlying disease. Iron malabsorption in patients with co-existing Crohn's disease exacerbates the ACD (65). Hemolytic anemia is a well-known phenomenon in Wilson's disease (66). Iron overload secondary to chronic hemolytic anemias might end up with liver cirrhosis (67). IDA is associated with celiac disease, a disease linked to increased risk of cirrhosis (68). Various medications cause anemia with different mechanisms. A rare side effect of azathioprine, a medication included in treatment of autoimmune hepatitis, is pure red cell aplasia (69). In a study by Patil et al, antiobacterial agents were reported to be associated with a hypocellular bone marrow in cirrhosis patients and cephalosporines were significantly linked to anemia (70). 


\section{Conclusion}

Anemia is a frequent finding in liver diseases. The condition is a sign of numerous various underlying mechanisms, some of which need investigation and specialized-treatment. IDA, ACD, hypersplenism and GI bleeding are the common etiologies of anemia in CLD. Disease- specific etiologies including viral induced aplastic anemia, malabsorption in inflammatory bowel diseases, hemolysis in Wilson's disease also contribute to exacerbation of the existing anemia. Table 2. summarizes the etiologies of anemia in CLD.

Table 2

Anemia in CLD and their characteristics

\section{Cause of anemia}

Portal hypertension

Viral infections

Alcohol

Anemia of Chronic disease

Bilirubin toxicity to RBCs

Medication side effects e.g. azathioprine, cephalosporines, interferon, ribavirin

Disease-specific

\section{Pathophysiology/Characteristic}

Dilutional low hematocrit count

GI bleeding

Hypersplenism

Cytokine induced aplastic anemia

Medication side effect

Malnutrition of B12 and folate

Spur cell anemia

Sideroblastic anemia

Alcohol toxicity to hematopoietic stem cells

Alcohol's interactions with folate metabolism

Chronic inflammatory states, cytokine induced

Eryptosis

Hemolysis

Red cell aplasia

Bone marrow suppression

Malabsorption

Hemolysis

\section{Abbreviations}


Anemia of Chronic Disease (ACD), Chronic Liver Disease (CLD), Gastrointestinal (GI), Hemoglobin (Hb), Hepatitis-Associated Aplastic Anemia (HAAA), Non-Alcoholic SteatoHepatitis (NASH), Iron Deficiency Anemia (IDA), Red Blood Cell (RBC)

\section{Declarations}

\section{Ethics approval}

The study was approved by the ethics committee of the department of Transplantation at Shiraz University of Medical Sciences.

\section{Consent for publication}

Not applicable.

\section{Availability of data and material}

Not applicable.

\section{Competing interests}

The authors declare no conflict of interest.

\section{Funding}

No funding was applied for this study.

\section{Authors' contributions}

MA searched the current literature and chose the articles to be cited and NA wrote the manuscript. Both authors assisted in preparing the manuscript and reviewed the paper and agreed to be accountable for all aspects of the work in ensuring that questions related to the accuracy or integrity of any part of the work are appropriately investigated and resolved.

\section{Acknowledgement}

Not applicable.

\section{References}

1. Singh S, Manrai M, V S P, Kumar D, Srivastava S, Pathak B. Association of liver cirrhosis severity with anemia: does it matter? Ann Gastroenterol. 2020;33(3):272-6.

2. Paternostro R, Kapzan L, Mandorfer M, Schwarzer R, Benedikt S, Viveiros A, et al. Anemia and iron deficiency in compensated and decompensated cirrhosis: Prevalence and impact on clinical outcomes. Journal of gastroenterology and hepatology. 2020;35(9):1619-27. 
3. Scheiner B, Semmler G, Maurer F, Schwabl P, Bucsics TA, Paternostro R, et al. Prevalence of and risk factors for anaemia in patients with advanced chronic liver disease. Liver International. 2020;40(1):194-204.

4. Bothou C, Rüschenbaum S, Kubesch A, Quenstedt L, Schwarzkopf K, Welsch C, et al. Anemia and Systemic Inflammation Rather than Arterial Circulatory Dysfunction Predict Decompensation of Liver Cirrhosis. Journal of clinical medicine. 2020;9(5).

5. Cai M, Yang N, Lin S, Ye B, Chen H, Mao WL. Anemia Predicts Poor Outcomes in Patients with HBVRelated Decompensated Cirrhosis. Clinical laboratory. 2021;67(3).

6. Yang J, Yan B, Yang L, Li H, Fan Y, Zhu F, et al. Macrocytic anemia is associated with the severity of liver impairment in patients with hepatitis $B$ virus-related decompensated cirrhosis: a retrospective cross-sectional study. BMC gastroenterology. 2018;18(1):161.

7. Kalaitzakis E, Josefsson A, Castedal M, Henfridsson P, Bengtsson M, Hugosson I, et al. Factors related to fatigue in patients with cirrhosis before and after liver transplantation. Clinical gastroenterology and hepatology : the official clinical practice journal of the American Gastroenterological Association. 2012;10(2):174-81, 81.e1.

8. Les I, Doval E, Flavià M, Jacas C, Cárdenas G, Esteban R, et al. Quality of life in cirrhosis is related to potentially treatable factors. European journal of gastroenterology \& hepatology. 2010;22(2):221-7.

9. Turner J, Parsi M, Badireddy M. Anemia. StatPearls [Internet]. 2021.

10. Farid Y, Bowman NS, Lecat P. Biochemistry, hemoglobin synthesis. 2019.

11. Haley K. Congenital Hemolytic Anemia. The Medical Clinics of North America. 2016;101(2):361-74.

12. Shah A. Hemolytic anemia. 2004.

13. Iwakiri Y. Pathophysiology of portal hypertension. Clin Liver Dis. 2014;18(2):281-91.

14. Ginès P, Fernández-Esparrach G, Arroyo V, Rodés J. Pathogenesis of ascites in cirrhosis. Seminars in liver disease. 1997;17(3):175-89.

15. Blendis L, Wong F. The hyperdynamic circulation in cirrhosis: an overview. Pharmacology \& Therapeutics. 2001;89(3):221-31.

16. Schrier RW. Water and sodium retention in edematous disorders: role of vasopressin and aldosterone. The American journal of medicine. 2006;119(7):S47-S53.

17. Hilzenrat N, Sherker AH. Esophageal Varices: Pathophysiology, Approach, and Clinical Dilemmas. International Journal of Hepatology. 2012;2012:795063.

18. Chapman J, Bansal P, Goyal A, Azevedo AM. Splenomegaly: StatPearls Publishing, Treasure Island (FL); 20202020.

19. Drevon L, Maslah N, Soret-Dulphy J, Dosquet C, Ravdan O, Vercellino L, et al. Anemia and hemodilution: analysis of a single center cohort based on 2,858 red cell mass measurements. Haematologica. 2021;106(4):1167-71.

20. Anand IS, Chandrashekhar Y, Ferrari R, Poole-Wilson PA, Harris PC. Pathogenesis of oedema in chronic severe anaemia: studies of body water and sodium, renal function, haemodynamic variables, 
and plasma hormones. British heart journal. 1993;70(4):357-62.

21. Cirera I, Elizalde JI, Pique JM, Feu F, Casadevall M, Goldin E, et al. Anemia Worsens Hyperdynamic Circulation of Patients with Cirrhosis and Portal Hypertension. Digestive Diseases and Sciences. 1997;42(8):1697-702.

22. Doan CA. Hypersplenism. Bulletin of the New York Academy of Medicine. 1949;25(10):625.

23. Lv Y, Gong X, Xie X, Wang B, Yang Y, Li Y. Clinical Study on the Relationship Between Hematocytopenia and Splenomegaly Caused by Cirrhotic Portal Hypertension. Cell Biochemistry and Biophysics. 2014;70(1):355-60.

24. Shah SH, Hayes PC, Allan PL, Nicoll J, Finlayson ND. Measurement of spleen size and its relation to hypersplenism and portal hemodynamics in portal hypertension due to hepatic cirrhosis. American Journal of Gastroenterology (Springer Nature). 1996;91(12).

25. Shah SH, Hayes PC, Allan PL, Nicoll J, Finlayson ND. Measurement of spleen size and its relation to hypersplenism and portal hemodynamics in portal hypertension due to hepatic cirrhosis. The American journal of gastroenterology. 1996;91(12):2580-3.

26. Tan $\mathrm{CH}$, Hall JA, Hammonds K, Dodlapati J, Linz WJ, Henderson SM. Relationship between splenomegaly and transfusion requirements in patients with cirrhosis. Proceedings (Baylor University Medical Center). 2020;34(1):44-8.

27. Lv Y, Yee Lau W, Wu H, Han X, Gong X, Liu N, et al. Causes of peripheral cytopenia in hepatitic cirrhosis and portal hypertensive splenomegaly. Experimental Biology and Medicine. 2017;242(7):744-9.

28. Kedia S, Goyal R, Mangla V, Kumar A, S S, Das P, et al. Splenectomy in cirrhosis with hypersplenism: improvement in cytopenias, Child's status and institution of specific treatment for hepatitis $\mathrm{C}$ with success. Annals of hepatology. 2012;11(6):921-9.

29. Zeng DB, Di L, Zhang RC, Guo QL, Duan BW, Jia CY, et al. The Effect of Splenectomy on the Reversal of Cirrhosis: a Prospective Study. Gastroenterology research and practice. 2019;2019:5459427.

30. Reverter J. Abnormal hemostasis tests and bleeding in chronic liver disease: are they related? Yes. Journal of Thrombosis and Haemostasis. 2006;4(4):717-20.

31. Mallet M, Rudler M, Thabut D. Variceal bleeding in cirrhotic patients. Gastroenterol Rep (Oxf). 2017;5(3):185-92.

32. Kalafateli M, Triantos CK, Nikolopoulou V, Burroughs A. Non-variceal Gastrointestinal Bleeding in Patients with Liver Cirrhosis: A Review. Digestive Diseases and Sciences. 2012;57(11):2743-54.

33. Khalifa A, Rockey DC. Lower Gastrointestinal Bleeding in Patients With Cirrhosis-Etiology and Outcomes. The American Journal of the Medical Sciences. 2020;359(4):206-11.

34. Canlas KR, Dobozi BM, Lin S, Smith AD, Rockey DC, Muir AJ, et al. Using Capsule Endoscopy to Identify GI Tract Lesions in Cirrhotic Patients With Portal Hypertension and Chronic Anemia. Journal of Clinical Gastroenterology. 2008;42(7). 
35. Alshaibani A, Dufour C, Risitano A, de Latour R, Aljurf M. Hepatitis-associated aplastic anemia. Hematology/Oncology and Stem Cell Therapy. 2020.

36. Rauff B, Idrees M, Shah SAR, Butt S, Butt AM, Ali L, et al. Hepatitis Associated Aplastic Anemia: A review. Virology Journal. 2011;8(1):87.

37. Langnas AN, Markin RS, Cattral MS, Naides SJ. Parvovirus B19 as a possible causative agent of fulminant liver failure and associated aplastic anemia. Hepatology. 1995;22(6):1661-5.

38. Ikawa Y, Nishimura R, Kuroda R, Mase S, Araki R, Maeba H, et al. Expansion of a liver-infiltrating cytotoxic T-lymphocyte clone in concert with the development of hepatitis-associated aplastic anaemia. British journal of haematology. 2013;161(4):599-602.

39. Ballard HS. Alcohol, bone marrow, and blood. Alcohol Research. 1993;17(4):310.

40. Halsted CH, Villanueva JA, Devlin AM, Chandler CJ. Metabolic interactions of alcohol and folate. The Journal of nutrition. 2002;132(8):2367S-72S.

41. Wiley JS, Moore MR. Heme biosynthesis and its disorders: porphyrias and sideroblastic anemias. 2000.

42. Holloway R. Sideroblastic anaemia secondary to chronic alcoholism: a case study and review. NEW ZEALAND JOURNAL OF MEDICAL LABORATORY SCIENCE. 2007;61(3):69.

43. Rock Jr WA, Stass SA. 1. Diagnostic Neuropathology, Harry V. Vinters, Michael A. Farrell, Paul S. Mischel, and Karl H. Anders.

44. Privitera G, Meli G. An unusual cause of anemia in cirrhosis: spur cell anemia, a case report with review of literature. Gastroenterology and hepatology from bed to bench. 2016;9(4):335.

45. Alexopoulou A, Vasilieva L, Kanellopoulou T, Pouriki S, Soultati A, Dourakis SP. Presence of spur cells as a highly predictive factor of mortality in patients with cirrhosis. Journal of gastroenterology and hepatology. 2014;29(4):830-4.

46. Ricard MP, Martínez ML, Ruiz J. Spur cell hemolytic anemia of severe liver disease. Haematologica. 1999;84(7):654-.

47. McHutchison JG, Manns MP, Longo DL. Definition and management of anemia in patients infected with hepatitis C virus. Liver International. 2006;26(4):389-98.

48. Lau JY, Tam RC, Liang TJ, Hong Z. Mechanism of action of ribavirin in the combination treatment of chronic HCV infection. Hepatology. 2002;35(5):1002-9.

49. De Franceschi L, Fattovich G, Turrini F, Ayi K, Brugnara C, Manzato F, et al. Hemolytic anemia induced by ribavirin therapy in patients with chronic hepatitis $\mathrm{C}$ virus infection: role of membrane oxidative damage. Hepatology. 2000;31(4):997-1004.

50. Ampuero J, Romero-Gómez M. Pharmacogenetics of ribavirin-induced anemia in hepatitis C. Pharmacogenomics. 2016;17(14):1587-94.

51. Abboud S, Haile DJ. A novel mammalian iron-regulated protein involved in intracellular iron metabolism. Journal of Biological Chemistry. 2000;275(26):19906-12. 
52. Ponka P, Beaumont C, Richardson DR, editors. Function and regulation of transferrin and ferritin. Seminars in hematology; 1998.

53. V AK, Rao PS, Adappa S, Balanthimogru P, Mahabala C. Correlation between serum ferritin and bone marrow iron stores. Tropical doctor. 2017;47(3):217-21.

54. Ganz T. Hepcidin. [Rinsho ketsueki] The Japanese journal of clinical hematology. 2016;57(10):19137.

55. Weiss G, Goodnough LT. Anemia of chronic disease. New England Journal of Medicine. 2005;352(10):1011-23.

56. Theurl I, Schroll A, Nairz M, Seifert M, Theurl M, Sonnweber T, et al. Pathways for the regulation of hepcidin expression in anemia of chronic disease and iron deficiency anemia in vivo. Haematologica. 2011;96(12):1761-9.

57. Handa P, Vemulakonda AL, Maliken BD, Morgan-Stevenson V, Nelson JE, Dhillon BK, et al. Differences in hepatic expression of iron, inflammation and stress-related genes in patients with nonalcoholic steatohepatitis. Annals of hepatology. 2017;16(1):77-85.

58. Gkamprela E, Deutsch M, Pectasides D. Iron deficiency anemia in chronic liver disease: etiopathogenesis, diagnosis and treatment. Ann Gastroenterol. 2017;30(4):405-13.

59. Weiss G, Ganz T, Goodnough LT. Anemia of inflammation. Blood. 2019;133(1):40-50.

60. Repsold L, Joubert AM. Eryptosis: An erythrocyte's suicidal type of cell death. BioMed research international. 2018;2018.

61. Lang K, Duranton C, Poehlmann H, Myssina S, Bauer C, Lang F, et al. Cation channels trigger apoptotic death of erythrocytes. Cell Death \& Differentiation. 2003;10(2):249-56.

62. Boas FE, Forman L, Beutler E. Phosphatidylserine exposure and red cell viability in red cell aging and in hemolytic anemia. Proceedings of the National Academy of Sciences. 1998;95(6):3077-81.

63. Lang E, Gatidis S, Freise NF, Bock H, Kubitz R, Lauermann C, et al. Conjugated bilirubin triggers anemia by inducing erythrocyte death. Hepatology. 2015;61(1):275-84.

64. Brito MA, Brites D. Effect of acidosis on bilirubin-induced toxicity to human erythrocytes. Molecular and Cellular Biochemistry. 2003;247(1):155-62.

65. Semrin G, Fishman DS, Bousvaros A, Zholudev A, Saunders AC, Correia CE, et al. Impaired intestinal iron absorption in Crohn's disease correlates with disease activity and markers of inflammation. Inflammatory bowel diseases. 2006;12(12):1101-6.

66. Ferenci P. Diagnosis of Wilson disease. Handbook of clinical neurology. 2017;142:171-80.

67. Hilgard P, Gerken G. Liver cirrhosis as a consequence of iron overload caused by hereditary nonspherocytic hemolytic anemia. World J Gastroenterol. 2005;11(8):1241-4.

68. Rubio-Tapia A, Murray JA. Liver involvement in celiac disease. Minerva Med. 2008;99(6):595-604.

69. Agrawal A, Parrott N, Riad H, Augustine T, editors. Azathioprine-induced pure red cell aplasia: case report and review. Transplantation proceedings; 2004: Elsevier. 
70. Patil A, Khillan V, Thakur M, Kale P, Bihari C. Antimicrobial-Induced Cytopenia and Bone Marrow Hypocellularity in Patients with Cirrhosis. Bone marrow research. 2018;2018:4029648. 